\title{
Relationship between time of initial diagnosis of acute myocardial infarction to primary angioplasty on patient's outcome
}

\author{
Marianna Saita RN, BSN, MSc루, George Argyriou RN, MSc, PhD², Olga Kadda RN, MSc, PhD², Dimitrios Protogiros RN, BSN, MSc', Polyxeni Detsi RN, \\ BSN $^{1}$, Georgia Toulia Lecturer ${ }^{5}$, Georgios Vasilopoulos Assistant Professor ${ }^{5}$, Christina Marvaki Emeritus Professor ${ }^{5}$
}

\begin{abstract}
Saita M, Argyriou G, Kadda O, et al. Relationship between time of initial diagnosis of acute myocardial infarction to primary angioplasty on patient's outcome. Curr Res Integr Med 2018;3(1):7-12.
\end{abstract}

OBJECTIVES: To explore the relationship between time of initial diagnosis of acute myocardial infarction to primary angioplasty on patient's outcome.

MATERIAL AND METHODS: A systematic review of published articles in international databases (CENTRAL, SCOPUS and MEDLINE). A time limited criterion was applied related to the articles publication (articles published between 2012 and 2016). Seventeen articles were found that met the criteria for participation in the review.

RESULTS: According to the results, the majority of studies showed that time to treatment (primary angioplasty) was less than 90 minutes from the initial assessment, resulting in better outcomes of patients.
The time from patient presentation to hospital in ST segment elevetion myocardial infarction (STEMI) diagnosis and laboratory activation for catheterization is a powerful guide to the total time from admittance to primary percutaneous coronary intervention (PPCI). In some of the stydies were described the effect of time from assessment on initiation of angioplasty in patients short-term or long-term outcomes and in others were investigated the reasons of delay until the onset of primary angioplasty. Endly in some were found time reduction practices from assessment to the onset of angioplasty and improving the clinical outcome.

CONCLUSION: Early treatment is important for the best clinical outcomes of patients with acute myocardial infarction (AMI) and seems to be feasible.

Key Words: Primary Percutaneous Coronary Intervention (PPCI); Door-To-Balloon (DTB); Acute myocardial infarction; Outcomes; Emergency Department (ED); Delay
A te myocardial infarction (AMI) remains a major cause of cardiovascular death in the world with morbidity and mortality rates remaining high (1). Although ST segment Elevation Myocardial Infarction (STEMI) mortality in-hospital has been significantly reduced with modern reperfusion therapies (primary angioplasty and thrombolysis) and secondary prevention has improved, overall mortality is unchanged since two thirds of patients die before their arrival in the hospital, mainly from deadly arrhythmias triggered by ischemia (2). Primary percutaneous coronary intervention (PPCI) is considered to play a central role in the treatment of STEMI patients, $(3,4)$. Previous studies have reported that early reperfusion may preserve more viable myocardium, which is associated with better outcomes for patients with STEMI myocardial infarction (5). Furthermore, have shown that reduced door-to-balloon (DTB) times improve mortality and clinical guidelines recommend that DTB time should be within 90 min (6). The American College of Cardiology/American Heart Association guideline and the European Society of Cardiology guidelines for STEMI recommends PPCI within 90 minutes of first medical contact $(7,8)$. Despite these guidelines and the development of regional STEMI systems of care, reperfusion goals are still not being achieved for most patients due to delays (9). This systematic review was designed to explore the relationship between time of initial diagnosis of acute myocardial infarction in an emergency department (ED) to primary angioplasty on patients outcome.

\section{METHODS}

To complete this bibliographic review, articles published over the last five years have been studied, namely 01/01/2012 to 31/12/2016. Electronic literature searches were conducted using the CENTRAL, SCOPUS and MEDLINE databases in January 2017. The review was conducted based on proposed benchmarks for a systematic review and meta-analyzes (PRISMA) (10). The index terms used in the search and the combination of these were: "primary percutaneous coronary intervention (PPCI)" OR "door-toballoon" AND "time" OR "acute myocardial infarction" OR "outcomes" OR "delay" and related to title search, summary, and keywords. Based on the title and summary of the articles resulting from the search, articles that could potentially be included in the review were identified after a detailed reading of the title and full text and in accordance with the inclusion criteria set. Furthermore, their literature was examined for the existence of relevant articles. Studies that followed quantitative methodology were included, while were excluded narrative or systematic reviews. The process of locating relevant articles for the review is summarized in the flow chart (Figure 1).

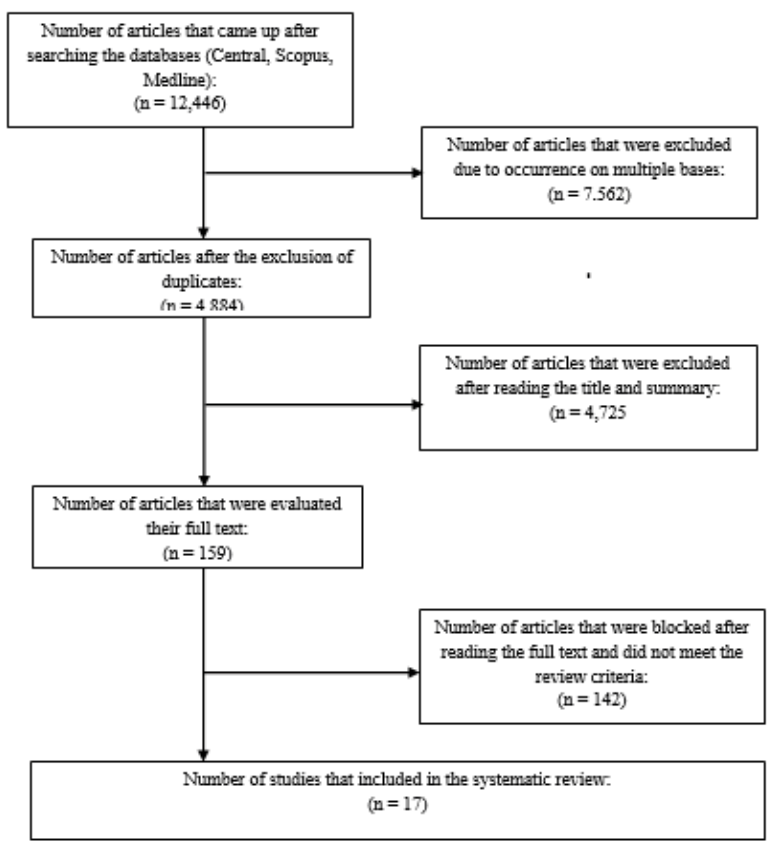

Figure 1 Flow chart for selecting the studies included in the review

${ }^{1}$ Department of Internal Medicine, Sismanogleio General Hospital of Athens, Greece, 2"Sotiria" General Hospital of Athens, Greece, ${ }^{3}$ Onassis Cardiac Surgery Center, Greece, ${ }^{4}$ Department of Nursing, School of Health Sciences, National and Kapodistrian University of Athens, Greece, ${ }^{5}$ Nursing Department, Technological Educational Institute, Athens, Greece

Correspondence: George Argyriou, "Sotiria" General Hospital of Athens, Greece, Telephone+306945386094, e-mail ageorge2000@yahoo.com

Received: April 19, 2018, Accepted: April 27, 2018, Published: April 30, 2018 
Saita et al.

\section{TABLE 1}

\section{Characteristics of review studies}

\begin{tabular}{|c|c|c|c|}
\hline $\begin{array}{c}\text { Author and } \\
\text { year }\end{array}$ & Study design & Studied sample & Aim \\
\hline $\begin{array}{c}\text { Shiomi et al., } \\
2012,(11)\end{array}$ & $\begin{array}{l}\text { Observation } \\
\text { study }\end{array}$ & $\begin{array}{l}3391 \text { patients with ST segment } \\
\text { elevation myocardial infarction } \\
\text { (STEMI) who had primary } \\
\text { percutaneous coronary } \\
\text { intervention within } 24 \text { hours of } \\
\text { symptom onset }\end{array}$ & $\begin{array}{l}\text { Evaluation of relation between } \\
\text { the time of symptoms onset } \\
\text { and the onset of primary } \\
\text { angioplasty and long term } \\
\text { clinical outcomes }\end{array}$ \\
\hline
\end{tabular}

A door to balloon time of less than 90 minutes was associated with a lower incidence of a composite of death and congestive heart failure in patients who presented within 2 hours of symptom onset $(11.9 \%(74 / 883)$ v $18.1 \%(147 / 655), \mathrm{P}=0.01$; relative risk reduction $34.3 \%)$ but not in patients who presented later $(19.7 \%(196 / 788) \vee 18.7 \%(135 / 1065)$, $\mathrm{P}=0.44 ;-5.3 \%$ )

The mean DTB time was $80.5 \mathrm{~min}(\mathrm{SD}=34.4$, median time $75 \mathrm{~min}$, range $30-195$ ). DTB time was $<90 \mathrm{~min}$ in $76.5 \%$, and DTB time $>90 \mathrm{~min}$ occurred in $23.5 \%$. Mean door to ECG $-6.5 \mathrm{~min}(\mathrm{SD}=2.7)$, mean time for the decision of $\mathrm{PCl}-7.5 \mathrm{~min}(\mathrm{SD}=10.5)$, mean time taken for the patient's consent - $19.6 \mathrm{~min}(\mathrm{SD}=17.6)$, for STEMI team activation - 6.7 $\min (S D=7.6)$, average time for financial process - $39.2 \mathrm{~min}(S D=22.9)$. Average time for sheath to balloon $-5.2 \mathrm{~min}(\mathrm{SD}=1.7)$. Hospital related delay occurred in $5 \%$, patient related delay in $80 \%$, both together in $15 \%$. Mortality among $<90$ min was $3.1 \%$, mortality among $>90$ min was $10 \%$

('p'=0.2)

237 adult patients with acute myocardial infarction Wang et al., Comparative (STEMI) who presented in 2012,(13) study the emergency department within 12 hours of the onset of symptoms

Median D2B time decreased from $107 \mathrm{~min}$ in the pre-on-site group to 72

Reduction of initiation time of $\mathrm{min}$ in the interim group, and to $47 \mathrm{~min}$ in the on-site group, respectively primary angioplasty in STEMI $(p<0.001)$. The percentage of D2B times $<90$ min increased from $34 \%$ patients by the application of to $78 \%$ and $96 \%$, respectively among the three groups $(p<0.001)$. Ina new care model hospital mortality did not differ significantly among the three groups $(4.8 \%, 2.2 \%$, and $6.1 \%$, respectively; $p=0.387)$

The median door-to-activation time was 19 minutes (interquartile range, 9-54). Variation in door-to-activation times explained $93 \%$ of the variation Evaluation of relation between in door-to-balloon times and demonstrated the strongest correlation with

Retrospective Registry data on 347 patients study of diagnosed with STEMI acute registries and underwent $\mathrm{PPCl}$ $\begin{array}{ccc}\text { McCabe et al., } & \text { medical myocardial infarction presented } \\ \text { 2012, (14) } & \text { and nursing to the emergency department }\end{array}$ $\begin{array}{ccc}\text { McCabe et al., } & \text { medical myocardial infarction presented } \\ \text { 2012, (14) } & \text { and nursing to the emergency department }\end{array}$ $\begin{array}{ccc}\text { McCabe et al., } & \text { medical myocardial infarction presented } \\ \text { 2012, (14) } & \text { and nursing to the emergency department }\end{array}$ $\begin{array}{ccc}\text { McCabe et al., } & \text { medical myocardial infarction presented } \\ \text { 2012, (14) } & \text { and nursing to the emergency department }\end{array}$

time of arrival at the hospital door-to-balloon times $(r=0.97)$. Achieving a door-to-activation time of $\leq$ to acute myocardial infarction 20 minutes resulted in an $89 \%$ chance of achieving a door-to balloon anosis with the initiation of primary angioplasty and assessment of factors affecting the activation times

time of $\leq 90$ minutes compared with only $28 \%$ for patients with a doorto-activation time $>20$ minutes. Factors significantly associated with door-to-activation time include the following: prehospital ECG use $(61 \%$ shorter, $95 \%$ confidence interval, -50 to $-72 \%$; $P<0.001$ ) and computed tomography scan use in the emergency department (245\% longer, 95\% confidence interval, +50 to $+399 \% ; P=0.001$ )

Of 410 patients with a suspected diagnosis of STEMI, $45(11 \%)$ underwent CT scanning before primary PCl. Only $2(4 \%)$ of these CT scans changed clinical management by identifying a stroke. Patients who Analysis the use of CT scan underwent CT scanning had far longer door-to-balloon times (median 166

Armstrong et al., Retrospective 410 STEMI patients underwent 2012, (23) study PPCl the emergency department prior to primary $\mathrm{PCl}$ and the investigation of related delays vs. 75 minutes, $\mathrm{p}<0.001)$ and higher in-hospital mortality (20\% vs. $7.8 \%$ $p=0.006$ ). After multivariate adjustment, CT scanning in the emergency department before primary $\mathrm{PCl}$ remained independently associated with longer door-to-balloon times (100\% longer, 95\% confidence interval 60 to $160, p<0.001$ ) but was no longer associated with mortality (odds ratio $1.4, p=0.5)$

12,146 cases of patients Swaminathan et Retrospective aged 18-90 with ST segment al., 2013, (15) study within 12 hours of symptom onset

Registration of non-system reasons of delayed initiation of angioplasty and their incidence on hospital mortality

Non-system delays occurred in $14.7 \%$ of patients $(n=12,146)$. Patients with non-system delays were more likely to be older, female, African American, and have greater comorbidities. The in-hospital mortality for patients treated without delay was $25 \%$ versus $15.1 \%$ for those with delay $(p<0.01)$. Non-system delay reasons included delays in providing consent $(4.4 \%)$, difficult vascular access $(8.4 \%)$, difficulty crossing the lesion (18.8\%), "other" (31\%), and cardiac arrest/intubation $(37.4 \%)$

Off -hours $\mathrm{PCl}$ occurred in $41 \%$ of patients. Medical contact to door time was similar in the Off -hour Group compared to the On-hour Group, $20.7 \pm 14.6$ versus $20.3 \pm 12.3$ min, respectively, $\mathrm{P}=0.47$. In patients with available data ( $n=774$ 1366), the door-to-catheterization laboratory with available data $(n=7741366)$, the door-to-catheterization laboratory
$(C L)$ activation time was signify- cantle shorter in the On-hour Group as compared to the Off -hour Group, $-4.9 \pm 11.9$ versus $-0.2 \pm 27.5 \mathrm{~min}$ respectively, $\mathrm{P}<0.0001$. Door-to-balloon time was significantly longer in the Off -hour Group compared to the On-hour Group, $74 \pm 35$ versus 60 \pm 26 min respectively, $P<0.0001$. Length of stay, vascular complications, achievement of TIMI 3 flow and in-hospital mortality were evaluated

With the use of currently available cardiovascular quality registries, it is unclear how many patients truly require unavoidable delays during primary $\mathrm{PCl}$. Patients with non-systems delay (NSD) had the worst outcomes regardless of centers for medicaid and medicare services

McCabe et al., Retrospective 456 STEMI patients underwent Determination of unavoidable 2014, (17) study PPCl delays in PPCl
Cotoni et al., Retrospective 43,909 registries of STEMI 2014, (18) study patients who underwent PPCI
Investigating the frequency of delays in PPCl application for nonsystem-related reasons and their impact on primary angioplasty initiation time (CMS) status. Between-registry agreement on patients with unavoidable delays was modest $(\mathrm{k}=0.32)$. Among CMS-included patients without NSD, $94 \%$ received $\mathrm{PCl}$ within 90 minutes compared with $29 \%$ of CMSexcluded patients with NSD $(P<0.001)$. Likewise, CMS-included patients without NSD had a 4-fold better 1-year mortality rate compared with CMS-excluded patients with NSD $(\mathrm{P}<0.001)$

Exclusion reasons included vascular access difficulty $(7.6 \%)$, cardiac arrest/intubation (38\%), and $\mathrm{PCl}$ procedural difficulties (20\%). Including patients with nonsystem delays significantly increased D2B times by $\leq$ 2 minutes for each group. The effect was larger on the proportion of patients having a $\mathrm{D} 2 \mathrm{~B} \leq 90$ minutes (low $83.6 \%$ to $85 \%$, intermediate $82.9 \%$ to $86.3 \%$, high $82 \%$ to $87.5 \%$, p $<0.001$, for all) 
Helve et al., Retrospective 500 STEMI patients underwent 2014, (19) comparative study $\mathrm{PCl}$ within 12 hours of the onset of symptoms
Determining recent trends in elays and factors associated with longer delays in $\mathrm{PCl}$ application
Anderson et al., Retrospective 2015, (9) study
33,901 cases of STEM patients underwent PPCI
Investigation of relation between direct transfer to the hemodynamic laboratory and expected outcomes of patients

52 STEMI patients who underwent PPCI were divided into 2 groups based on whether they presented before and hospital design on PPC (21) or after (31) the opening of application time in STEMI the Interventional platform (IP) patients of a new medical center

793 STEMI patients who underwent PPCl off-hours Brown et al., Retrospective were categorized into 2 2015, (6) study groups, pre-introduction (295) and post- introduction (498) of the protocol
Assessment of the effect of protocol change from phone call to simultaneous computerized alert in time from PPCl admittance, hospital treatment and 1year mortality

Delays of the emergency medical system (EMS) were longer in HUSSTEMI I than II (medians 81 vs. $67 \mathrm{~min}$, respectively, $p<0.001$ ). Although door-to-balloon times were longer in the later dataset (33 vs. $48 \mathrm{~min}$ $\mathrm{p}<0.001)$ most of the patients $(75.3 \%$ vs. $62.8 \%$, respectively, $\mathrm{p}=0.010)$ were treated within the recommendation ( $<60 \mathrm{~min}$ ) of the European Society of Cardiology (ESC). In HUS-STEMI II, patient arrival at the hospital during off-hours was associated with longer door-to-balloon time (40 and $57.5 \mathrm{~min}, \mathrm{p}=0.001)$ and system delay $(111$ and $127 \mathrm{~min}$ $\mathrm{p}=0.009$ ). However, in HUS-STEMI I, arrival time did not impact the delays. Longer system delay was associated with higher mortality rates.

The majority of patients were transferred directly to the cath lab $(26,510$ $(78.2 \%))$, and 7,391 patients $(21.8 \%)$ were transferred first to the hospital emergency department/ward. Compared with patients transferred first to the emergency department/ward, STEMI patients transferred to the cath lab had significantly lower first door-to-balloon times (median 191 versus 116 minutes, $P<0.0001$ ). After multivariable logistic regression, patients transferred directly to the cath lab also had lower adjusted mortality risk (odds ratio $0.58,95 \%$ confidence interval $0.51-0.66, \mathrm{P}<0.0001$ ). Cardiogenic shock, heart failure signs/symptoms, and nonsystem reasons for reperfusion delay were present in $11 \%, 15 \%$, and $28 \%$ of patients transferred first to the emergency department/ward, respectively This study showed that the new hospital design had significant effects on immediate patient care by improving the DTB time. The mean DTB time significantly improved by 11.7 minutes after the opening of the IP $(P=0.016)$, and all cases had a DTB time 90 minutes or less as compared with $90.4 \%$ prior. Eighty-nine percent of the overall improvement in DTB happened before the patient reached the catheterization table. Important factors were the new emergency department (ED) design that facilitates rapid patient triage and the direct connection between the ED and cath lab

Out of hours $(\mathrm{OOH}) \mathrm{PPCl}$ was performed for 793 patients (mean age $61,73.4 \%$ male) - 295 in Group 1 and 498 in Group 2. Median DTB times were 92 min (interquartile range (IQR) 75-111) for Group and 76 min (IQR 64-97) for Group 2 ( $p<0.0001)$. Forty-eight percent achieved DTB in $\leq 90 \mathrm{~min}$ in Group 1 compared to $70 \%$ in Group 2 ( $p$ 0.0001). Computerized alert was associated with a shorter DTB time on multivariate analysis (beta coefficient $-0.09, p=0.03$ for linear regression and $\mathrm{OR} 2.8,95 \% \mathrm{Cl} 1.6-5.0, \mathrm{p}<0.0001$ for logistic regression). In hospital mortality was $4.1 \%$ in Group 1 and $5 \%$ in Group $2(p=0.60)$. All-cause mortality at 1 year was $6.1 \%$ in Group 1 and $9.9 \%$ in Group 2 ( $p=0.09)$.

Patients were divided into 3 groups according to culprit artery location right coronary artery (RCA), left anterior descending artery (LAD), and left circumflex artery (LC), and associations with door-to-balloon time (DBT) were assessed. The LC group had a trend toward a longer DBT among the 3 groups ( 97.1 (RCA) vs 98.1 (LAD) vs 105.1 (LC) minutes; $p=0.058$ ).

Evaluation of the effect of culprit artery location on Kuno et al., Retrospective 1,725 cases of STEMI patients 2015, (24) study underwent PPCI door-to-balloon time of STEM patients who underwent $\mathrm{PC}$ left coronary artery lesion (3.5\% (RCA) vs $6.3 \%$ (LAD) vs $5.4 \%$ (LC) $p=0.041)$. In-hospital mortality for patients with DBT $>90$ minutes was significantly higher compared with patients with DBT $\leq 90$ minutes $(6.5 \%$ vs $3.6 \% ; p=0.006$ ). Multivariate logistic regression analysis revealed that the LC location was an independent predictor for DBT >90 minutes (odds ratio, $1.45 ; 95 \%$ confidence interval, 1.04 to 2.01 ; $p(0.028)$

Determination of optimal culprit-vesse revascularization strategy before contralateral or complete diagnostic angiography correlated with a reduction in delays and outcomes of STEMI patients

Investigation of the age dependent impact of the superfast door-to-balloon times $\leq 60$ minutes as recommended by the new ESC guideline for STEMI patients undergoing $(\mathrm{PPCl})$

3,877 cases of STEMI patients who underwent $\mathrm{PPCl}$ were stratified into 3 groups: $<6$

Song et al., Retrospective hours from onset of symptoms 2016, (21) study to PPCI application $(2,120)$ $6-12$ hours $(1,266)$ and $>12$ hours (491)
Performing culprit-vessel primary percutaneous coronary intervention before contralateral or complete diagnostic angiography is associated with a statistically significant reduction in vascular access-to-balloon time, although the 4- to 6-minute difference is unlikely to be clinically relevant. This small but significant reduction could translate in an augmentation in the proportion of patients treated within recommended delays. However, there was no significant difference in 30-day clinical outcomes associated with these delays reduction

In patients aged $<65$, the mortality rate decreased linearly with D2B time shortening (>90 $\mathrm{min}$ vs. $61-90 \mathrm{~min}$ vs. $\leq 60 \mathrm{~min}=14.9 \%$ vs. $13.3 \%$ vs. $1.2 \%, \mathrm{P}=0.001)$. Contrarily, shortening of $\mathrm{D} 2 \mathrm{~B}$ time was not associated with reduced mortality rate in patients aged $\geq 65$ ( $>90 \mathrm{~min}$ vs. $61-90 \mathrm{~min}$ vs. $\leq 60 \mathrm{~min}=23.5 \%$ vs. $19 \%$ vs. $18.9 \%, P=0.99$ ). In younger patients but not the elderly, a D2B time of $<60 \mathrm{~min}$ has sufficient power to predict mortality with a sensitivity of 0.83 , specificity of 0.74 , and Youden index of 0.57

The median of symptom-onset-to-balloon (S2B) time was 5.5 (3.75 to 8.50) hours. Longer S2B time was associated with higher in-hospita mortality ( $<6$ hours: $2.7 \%$; 6 to 12 hours: $3.4 \%$; $>12$ hours: $4.9 \%$; $\mathrm{p}=0.047)$ and ST-segment resolution $<50 \%(<6$ hours: $16.7 \% ; 6$ to 12 hours: $19.2 \%$; >12 hours: $24.3 \% ; p=0.002$ ) but not with major adverse cardiovascular and cerebrovascular events (MACCE). Longer (S2B) time was associated with impaired myocardial perfusion but not with inhospital mortality or MACCE 
The number of articles resulted from the search on the bases CENTRAL, SCOPUS, MEDLINE were 12.446. From examining titles and abstracts, 159 articles were considered likely to be included. Of these, 17 met the inclusion criteria for this review. Of the studies that were excluded, the most of them did not evaluate the effect of time from the onset of primary angioplasty to the final outcome in patients with acute myocardial infarction and an article was reported in a study protocol.

\section{RESULTS}

The characteristics of the review studies are summarized in Table 1. In all 17 studies that met the inclusion criteria and were used in the review, the majority of these were conducted in the United States $(n=8,47,06 \%)$, while the others in Japan $(\mathrm{n}=2,11.76 \%)$, Taiwan $(\mathrm{n}=2,11.76 \%)$, and one in Canada (5.88\%), United Kingdom (5.88\%), India (5.88\%), Finland (5.88\%) and China (5.88\%). Over the period 2012-2016, which was a search criterion of the studies, five studies were published in 2012 (29.41\%), two in $2013(11.76 \%)$, three in $2014(17.65 \%)$, three in $2016(17.65 \%)$ and four in $2015(23,53 \%)$. With regard to the methodological design of the studies, most $(n=12,70.59 \%)$ were retrospective and were based on data and recordings of medical records of hospital and cardiovascular records of hospitals and/or cardiology companies, while the remaining five were crosssectional observational studies.

Investigation the correlation of time from initial assessment to emergency department until the onset of primary angioplasty with the final outcome in patients with acute myocardial infarction was evaluated in all studies included in the review. In majority of cases, primary angioplasty was achieved initiation time of less than 90 minutes from the initial assessment, resulting in better outcomes of patients $(5,6,9,11-21)$. However, in case of performing culprit-vessel primary percutaneus coronary intervention before the unilateral or complete diagnostic angiography is associated with a statistically significant reduction in vascular access-to-balloon time, although the difference of 4-6 minutes is unlikely to be clinically relevant (22). The time from patient presentation to hospital in STEMI myocardial infarction diagnosis and laboratory activation for catheterization is a powerful guide to the total time from admittance to PPCI (14).

Furthermore, to the case where a computed tomography (CT) scan was applied prior to PCI application (23) and when the stenosis site was investigated (24), in the other studies, an average angioplasty initiation time of less than 90 minutes was observed in the majority of patients. In some of them also were described the effect of time from assessment on initiation of angioplasty in patients short-term or long-term outcomes and in others were investigated the reasons of delay until the onset of primary angioplasty. Endly in some were found time reduction practices from assessment to the onset of angioplasty and improving the clinical outcome.

Effect of time between assessment and angioplasty onset on patient outcomes

The studies that evaluated the impact of time from the initial assessment on emergency department until the onset of primary angioplasty, showed better direct and long-term clinical outcomes for patients with AMI STEMI diagnosis, which were submitted to PPCI within the indicated times. In particular, patients had better triennial outcome, with lower rates of composite death and congestive heart failure (11), lower probability of death in hospital (15) and lower overall mortality rates $(9,12,19,21)$. In addition, the longest time was associated with disturbed myocardial perfusion but not with in-hospital mortality or with significant adverse cardiovascular and cerebrovascular events or other morbidity in the hospital (21). However, the survival rates of patients on early angioplasty appear to be associated with younger age (less of 65 years) (5), as well as the stenosis at which patients with lesion in the left anterior descending artery appeared higher mortality rates (24). Furthermore, delays which are not due to the health system organizational structure appear to be responsible for a 4 -fold higher mortality rate of one year (17), while delayed PCI application off time hemodynamic laboratory operating did not appear to adversely affect hospitalization time, vascular complications, thrombolysis in myocardial infarction (TIMI) 3 final flow and in-hospital mortality (16). However, benefits were only limited to the patients who presented to emergency department early after the onset of symptoms (11).

Reasons of delay until the onset of primary angioplasty

The results of the studies that evaluated the reasons for delaying PPCI application have shown that they may be related to either patients, health professionals or both. The electrocardiogram implementation time (ECG) $(12,14)$, decision making on PCI, patient consent $(12,15)$, activation of the catheterization group, economic processes (12), the use of axial tomography in the emergency department $(14,23)$ are agents of delay in initiating primary angioplasty. Furthermore, other reasons found are difficulty in vascular access, difficulty passing through the lesion, cardiac failure / intubation and "other reasons" $(9,15,18)$. Moreover, the characteristics of patients predisposing for delays in the onset of primary angioplasty appear to be older, female sex, most co-morbidities and African-American patients (15). The delays due to the health system organizational structure seem to decrease over time (19), however, the longer time from occurrence of symptoms until the attendance to emergency department affected the immediate onset of angioplasty (21) Lastly, a patient off time attendance was sometimes associated with a PCI initiation delay (19) and other times not (12).

Reduction practices of the time from estimation to the onset of primary angioplasty and improvement in clinical outcome

In some of the review studies, additional practices have been evaluated to help reduce the initial angioplasty start time, with the ultimate goal of improving clinical outcomes for patients. First, architectural design of hospitals seems to have a significant impact on patient care, reducing time from initial assessment to catheterization in cases where rapid patient classification is facilitated, and a direct link between emergency department and the catheterization laboratory is achieved (20). Direct transfer of patients with STEMI to the laboratory was associated with significantly lower primary angioplasty initiation times, faster reperfusion, and lower mortality risk in comparison with the first transfer to the emergency department (9). Another study showed that the establishment of a cardiological approach by a team of health professionals in the ED reduced the time of primary angioplasty in patients with STEMI (13). Also, the activation time of the laboratory to initiate post-evaluation PPCI appears to be lower at $8 \mathrm{am}-5 \mathrm{pm}$ (16), but a simultaneous electronic alert for the off-hours of the catheterization laboratory may reduce the time from entering PPCI and increasing the number of patients achieving target times (6) The performance of PPCI prior to contralateral or complete diagnostic angiography is associated with a statistically significant reduction in vascular access time (22), but not performing CT prior to primary PCI that changes clinical management in a very small percentage of patients (23).

\section{DISCUSSION}

This systematic bibliographic review examined key questions that are important in understanding the recent findings on the delay from the initial assessment in the emergency department to the onset of primary angioplasty of patients with AMI. The main messages are as follows: 1. the improvement in the care of AMI STEMI patients has significantly contributed to the reduction of time from patient admittance to hospital to catheterization laboratory; 2 . longer waiting time to PPCI application adversely affects shortterm and long-term clinical outcomes of patients; 3. factors that prolong waiting time can be categorized according to the characteristics of the patients, the characteristics of the group of health professionals and the care procedures and the characteristics of the hospitals/care system; and 4. Good "time-reduction practices have been described to initiate primary angioplasty from the time of assessment.

The incidence of coronary heart disease remains high and the disease is responsible for more than 7 million deaths worldwide annually (25). Despite the decline in mortality, the time for presentation acute myocardial infarction patients into care is still a major public health problem (26). Time until the onset of treatment is an essential predictor of morbidity and mortality in patients with acute ST segment elevation (STEMI) myocardial infarction and are about to undergo pharmacological or mechanical reperfusion therapy (27). Furthermore, delay in treatment reduces the effectiveness of interventions and leads to negative outcomes (28).

Guidelines suggest that treatment commences within no more than 90 minutes from the onset of symptoms (25). Significant hospital delays in treatment are responsible for up to $40 \%$ of patients not achieving the target (29). Approximately one in two AMI cases are fatal before admittance in hospital and occur more frequently within one hour of the onset of symptoms (26), whereas delayed onset of primary angioplasty by over two hours increases mortality (29). This review showed that in most cases primary angioplasty was achieved start within 90 minutes from the initial assessment, resulting in better outcomes of patients and agreement with the target time of guidelines $(5,6,9,11-21)$ 
Furthermore, from the review studies it appeared that the immediate and outpatient outcomes of the patients were better when the time from the initial assessment in the emergency department to the catheterization laboratory was within the indicated targets. In particular, lower rates of in-hospital mortality, fewer complications, and higher survival rates were recorded up to three years after surgery $(9,11,12,15,19,21)$. Prognostic factors of delayed initiation of primary angioplasty include hospital transport time, no search for care, admittance to a non-specialized center (27), and not existence of angina pain (30). The findings of this review also show that patient-related factors (age, female gender, co-morbidity such us diabetes and obesity), PCI consent, failure to identify symptoms and delayed search for care $(12,15,21)$ appear to be those that eventually increase the time to the initiation of primary angioplasty. Other prognostic factors have been found to concern the system (19) and the health care professionals $(9,12,14,15,18)$.

In addition, from the studies used in this review some good practices appeared to reduce the waiting time from assessment in primary angioplasty $(9,20)$. These practices include that the catheterization laboratory is closer to the emergency department site, resulting in immediate patient transfer and PCI application. Furthermore, admittance to hospital non-working hours at the hemodynamic laboratory increases the PCI initiation time (16), while cardiac evaluation by a specialized team in the emergency department area improves clinical times (13). Understanding the time-related factors from assessment to primary angioplasty can lead to an improvement in the quality of care for STEMI patients. Primary PCI appears to be the most effective reperfusion therapy for patients with STEMI (29).

\section{LIMITATIONS}

Study selection criteria as well as search results create some limitations on this review. Initially, one of the limitations is the small number of studies selected and in particular 17 studies in their entirety. Another limitation is the quality of the methodology of published studies. There are particular challenges that have been described for systematic reviews, especially when retrospective studies or observational studies are used and for prognostic factor reviews. A limitation on the systematic reviews to be considered in this paper is related to the methodology of the studies that included in review results. According to the Cochrane Systemic Reviews Manual (31) it is noted that in literature reviews including observational studies or retrospective studies the quality and generalization of results is a major problem than with the reviews of randomized clinical tests. In this review, there is a remarkable absence of randomized trials for the subject to be studied as well as the search did not result in such studies. Furthermore, retrospective studies are likely to lead to a positive confusion about the factor or intervention studied, as most reports describe improvement in time from evaluation to PCI over the years. Endly, there is also a limitation of no unpublished bibliography was searched.

\section{CONCLUSION}

This review has shown that studies which were correlating time from initial evaluation to the onset of primary angioplasty and final outcome in patients with AMI, have highlighted the importance of early treatment, which is feasible in the majority of cases. The time from evaluation to catheterization includes only a small part of the overall delay for treatment and the overall delay of the healthcare system. However, a delay of over 90 minutes leads to difficult clinical outcomes, the range of which varies from complications in catheterization or post-surgery, worse direct and distant clinical outcomes, to death in hospital. Many factors have been reported to affect the time from evaluation to the onset of PCI in patients with STEMI who have undergone primary angioplasty. The overall quality of evidence for these factors is low and is largely based on observational studies or retrospective studies. Furthermore, systematically recording the results has highlighted the characteristics that can urge health professionals to reconsider the methods they use to treat patients with AMI in an emergency department. The reasons that increase the time for primary angioplasty are distinguished by system factors, the patient and health professionals. Clinical use of these findings could help treat patients more effectively and improve outcomes.

\section{REFERENCES}

1. Whindecker S, Kolh P, Alfonso F, et al. 2014 ESC/EACTS Guidelines on myocardial revascularization The Task Force on Myocardial Revascularization of the European Society of Cardiology (ESC) and the European Association for Cardio-Thoracic Surgery (EACTS) Developed with the special contribution of the European Association of Percutaneous Cardiovascular Interventions (EAPCI). Eur Heart J 2014;35(37):2541-619.

2. Goudevenos G. Annotation in the European Society of Cardiac Society (ESC) 2017 Newest Guidelines for Acute Myocardial Infarction (STEMI). 2017.

3. Gibson C, Pride Y, Frederick P, et al. Trends in reperfusion strategies, door-to-needle and door-to-balloon times, and in-hospital mortality among patients with ST-segment elevation myocardial infarction enrolled in the national registry of myocardial infarction from 1990 to 2006. Am Heart J 2008;156(6):1035-44.

4. Eagle KA, Nallamothu BK, Mehta RH, et al. Trends in acute reperfusion therapy for ST-segment elevation myocardial infarction from 1999 to 2006: we are getting better but we have got a long way to go. Eur Heart J 2008;29(5):609-17.

5. Wang YC, Huang YY, Lo PH, et al. Age-dependent impact of new ESCGuideline recommended door-to-balloon times on mid-term survival in acute ST-elevation myocardial infarction patients undergoing primary percutaneous coronary intervention. Int J Cardiol 2016;222:242-6.

6. Brown RA, Varma C, Connolly DL, et al. Simultaneous computerised activation of the primary percutaneous coronary intervention pathway reduces out-of-hours door-to-balloon time but not mortality. Int J Cardiol 2015;186:226-2.

7. Kushner FG, Hand M, Smith SC Jr, et al. 2009 focused updates: ACC / AHA guidelines for the management of patients with ST-elevation myocardial infarction (updating the 2004 guideline and 2007 focused update) and ACC / AHA / SCAI guidelines on percutaneous coronary intervention (updating the 2005 guideline and 2007 focused update) a report of the American College of Cardiology Foundation / American Heart Association Task Force on Practice Guidelines. Circulation 2009;120(22):2271-306.

8. Van de Werf F, Bax J, Betriu A, et al. Management of acute myocardial infarction in patients presenting with persistent ST-segment elevation. The task force on the management of ST-segment elevation acute myocardial infarction of the European Society of Cardiology. Eur Heart J 2008;29(23):2909-45.

9. Anderson LL, French WJ, Peng A, et al. Direct transfer from the referring hospitals to the catheterization laboratory to minimize reperfusion delays for primary percutaneous coronary intervention: insights from the national cardiovascular data registry. Circ Cardiovasc Interv 2015;8(9):e002477.

10. Moher D, Liberati A, Tetzlaff J, et al. Preferred Reporting Items for Systematic Reviews and Meta-Analyses: The PRISMA Statement. PLoS Med 2009;6(7): e1000097.

11. Shiomi H, Nakagawa Y, Morimoto T, et al. Association of onset to balloon and door to balloon time with long term clinical outcome in patients with ST elevation acute myocardial infarction having primary percutaneous coronary intervention: observational study. BMJ 2012;344: e3257.

12. Victor SM, Gnanaraj A, Vijayakumar S, et al. Door-to-balloon: Where do we lose time? Single centre experience in India. Indian Heart Journal 2012;64(6):582-7.

13.Wang, YC, Lo PH, Chang SS, et al. Reduced door-to-balloon times in acute ST-elevation myocardial infarction patients undergoing primary percutaneous coronary intervention. Int J ClinPract 2012;66(1):69-76.

14. McCabe JM, Armstrong EJ, Hoffmayer KS, et al. Impact of doorto-activation time on door-to-balloon time in primary percutaneous coronary intervention for st-segment elevation myocardial infarctions: A report from the activate-sf registry. Circ Cardiovasc Qual Outcomes 2012;5(5):672-9.

15. Swaminathan RV, Wang TY, Kaltenbach LA, et al. Nonsystem reasons for delay in door-to-balloon time and associated in-hospital mortality: A report from the national cardiovascular data registry. J Am Coll Cardiol 2013;61(16):1688-95.

16. Shavelle DM, Zheng L, Ottochian M, et al. Time of day variation in door 
to-balloon time for STEMI patients in Los Angeles County: Does time of day make a difference?, Acute Cardiac Care 2013;15(3):52-7.

17. McCabe JM, Kennedy KF, YehRW. Defining unavoidable delays in primary percutaneous coronary intervention: discordance among patients excluded from national cardiovascular quality registries. J Am Heart Assoc 2014;3(3): e000944

18. Cotoni DA, Roe MT, Li S, et al. Frequency of non-system delays in ST-elevation myocardial infarction patients undergoing primary percutaneous coronary intervention and implications for door-to-balloon time reporting. Am J Cardiol 2014;114(1);24-28.

19. Helve S, Viikila J, Laine M, et al. Trends in treatment delays for patients with acute ST-elevation myocardial infarction treated with primary percutaneous coronary intervention. BMC Cardiovascular Disorder 2014;14(1):115.

20.Poulin MF, Appis A, Purim-Shem-Tov Y, et al. Impact of a novel interventional platform and hospital design on the door-to-balloon time in patients presenting with ST-segment elevation myocardial infarction. Crit Pathways in Cardiol 2015;14(1):39-43.

21.Song F, Yu M, Yang J, et al. Symptom-onset-to-balloon time, ST-segment resolution and in-hospital mortality in patients with ST-segment elevation myocardial infarction undergoing primary percutaneous coronary intervention in China: from China acute myocardial infarction registry. Am J Cardiol 2016;118(9):1334-9.

22. Couture EL, Berube S, Dalery K, et al. Culprit vessel revascularization prior to diagnostic angiography as a strategy to reduce delays in primary percutaneous coronary intervention: A propensity-matched analysis. Circ Cardiovasc Interv 2016;9(5): e003510.

23. Armstrong EJ, Kulkarni AR, Hoffmayer KS, et al. Delaying primary percutaneous coronary intervention for computed tomographic scans in the emergency department. Am J Cardiol 2012;110(3):345-9.
24. Kuno T, Kohsaka S, Numasawa Y, et al. Location of the culprit coronary lesion and its association with delay in door-to-balloon time. Am J Cardiol 2015;115(5):581-6.

25.Levine GN, Bates ER, Blankenship JC, et al. 2015 ACC/AHA/SCAI Focused update on primary percutaneous coronary intervention for patients with ST-elevation myocardial infarction: An Update of the 2011 ACCF/AHA/SCAI guideline for percutaneous coronary intervention and the $2013 \mathrm{ACCF} / \mathrm{AHA}$ guideline for the management of ST-elevation myocardial infarction. J Am Coll Cardiol 2016;67(12):1235-50.

26.Johansson I, Strömberg A, Swahn E. Ambulance use in patients with acute myocardial infarction. J Cardiovasc Nurs 2004;19(1):5-12.

27.Park JS, Cha KS, Shin D, et al. Impact of none chest pain complaint as a presenting symptom on door-to-balloon time and clinical outcomes in patients with acute ST-elevation myocardial infarction. Am J Cardiol 2014;114(12):1801-9.

28.Zegrean M, Fox-Wasylyshyn SM, El-Masri MM. Alternative coping strategies and decision delay in seeking care for acute myocardial infarction. J Cardiovasc Nurs 2009;24(2):151-5.

29. Lubovich A, Hamood H, Behar S, et al. Bypassing the emergency room to reduce door-to-balloon time and improve outcomes of patients with ST elevation myocardial infarction: The acute coronary syndrome israeli survey experience. Isr Med Assoc J 2011;13(4):216-9.

30. DeVon HA, Hogan N, Ochs AL, et al. Time to treatment for acute coronary syndromes: The cost of indecision. J Cardiovasc Nurs 2010;25(2):106-14

31. Higgins J, Green S. Cochrane handbook of systematic reviews of interventions. West Sussex, UK: John Wiley \& Sons Ltd, West Sussex, UK. 2008. 